\title{
Auction-Style Cooperative Medium Access Control
}

\author{
J. Feng, R. Zhang and L. Hanzo \\ School of ECS., Univ. of Southampton, SO17 1BJ, UK. \\ Tel: +44-23-80-593 125, Fax: +44-23-80-593045 \\ Email: 1h@ecs.soton.ac.uk, http://www-mobile.ecs.soton.ac.uk
}

\begin{abstract}
A cooperative Medium Access Control (MAC) protocol employing auction-style relay selection combined with superposition coding is designed. In our proposed scheme, the source achieves its target throughput with the aid of cooperation. The potential candidate relays carry out autonomous decisions concerning whether to contend for a transmission opportunity to convey their traffic in the light of their individual energy requirements. Simulation results demonstrate that our scheme is capable of providing both considerable energy savings, as well as substantial throughput improvements for both the source and the relay in a 'win-win' cooperation regime.
\end{abstract}

\section{INTRODUCTION}

It is important to design appropriate Medium Access Control (MAC) protocols for supporting cooperative physical layer techniques. Most current cooperative MAC protocols were designed for either maximizing the throughput [1] or minimizing the energy consumption [2], [3]. By contrast, both Zhao et al. [4] and Shirazi et al. [5] designed meritorious algorithms for improving the achievable throughput, while reducing the energy consumption imposed. However, the above-mentioned cooperative MAC protocols were developed on the basis of the common assumption that the relays agree to altruistically forward the data frames of the source.

In order to consider the selfish behavior of the mobile relays, Stanojev et al. [6] proposed an auction-based cooperative Automatic Repeat reQuest (ARQ) scheme relying on a socalled spectrum-leasing paradigm. However, the attainable energy efficiency was not considered in this cooperative ARQ scheme. As a further advance, Mukherjee et al. [7] developed an auction-theoretic cooperative partner selection scheme for striking a tradeoff between the attainable throughput and energy efficiency. However, the potentially corrupted data received from the direct transmission link was not actively exploited. Furthermore, no particular transmission frame structure and signaling procedures were designed in [6], [7].

Against the above background, this paper proposes a cooperative MAC-layer protocol for a network supporting the source with the aid of relays for the sake of minimizing the total energy consumption and for improving the source's throughput, while simultaneously conveying the relay's own traffic. The main distinguishing aspects of our protocol are:

1) The proposed cooperative MAC-layer protocol benefiting from auction-style single relay selection is developed for striking a tradeoff between the achievable throughput

The financial support of the RC-UK under the auspices of the UKIndia Advanced Technology Center in Wireless Communications as well as that of the EPSRC under the China-UK Science Bridge in $4 \mathrm{G}$ wireless communications is gratefully acknowledged. and energy efficiency for both the source and relay in a practical network environment.

2) Specifically, we design a new superposition coded solution for the relays to joint encode the source's and relay's data, in order to satisfy the relay's service requirement.

The rest of this paper is organized as follows. The system model and the details of our protocol design are described in Section II. Section III introduces the advocated philosophy of auction-style single relay selection scheme, which is followed by the description of our direct and relayed frame combining strategy in Section IV. In Section V we quantified the attainable performance. Finally, we conclude in Section VI.

\section{System MODEL}

\section{A. Network Construction and Assumptions}

As seen in Fig 1, we consider a cooperative network having a primary single source $\mathcal{S}$ and a total of $N$ relays in the set $\mathcal{R}=\left\{\mathcal{R}_{1}, \ldots, \mathcal{R}_{N}\right\}$, as well as a common destination $\mathcal{D}$, where the $N$ relays act as secondary sources and $\mathcal{D}$ may be a Base Station (BS) or an ad hoc cluster head. In order to improve both the throughput and energy efficiency attained by the system, $\mathcal{S}$ invites the best relay candidate to cooperate in a manner that they both have an opportunity to transmit their buffer-content at a low total energy consumption. In turn, all the relay nodes (RNs) that have data in their buffer would compete for a transmission opportunity in the auction-style relay selection scheme to be introduced. Finally, $\mathcal{D}$ combines the direct transmission and the superposition-coded relayed transmission. We made the following assumptions:

1) All the channels involved are assumed to undergo quasistatic fading, hence the complex-valued fading envelope remains constant during a transmission burst, while it is faded independently between the consecutive transmission bursts. Within a given transmission burst, the duplex bi-directional channels between a pair of actively communicating nodes are assumed to be identical, while the channels of any of the remaining links are independent.

2) We assume perfect channel estimation for all nodes concerning their own channels, but no knowledge of the remaining links is assumed.

3) We consider the combined effects of flat Rayleigh fading as well as free-space pathloss that is modeled by $\rho=$ $\lambda^{2} / 16 \pi^{2} d^{\eta}$, where $\lambda$ represents the wave-length, $d$ is the transmitter-to-receiver distance and $\eta=2$ denotes the pathloss exponent. All nodes are assumed to be limited by the same maximum transmit power $P_{\max }$. 


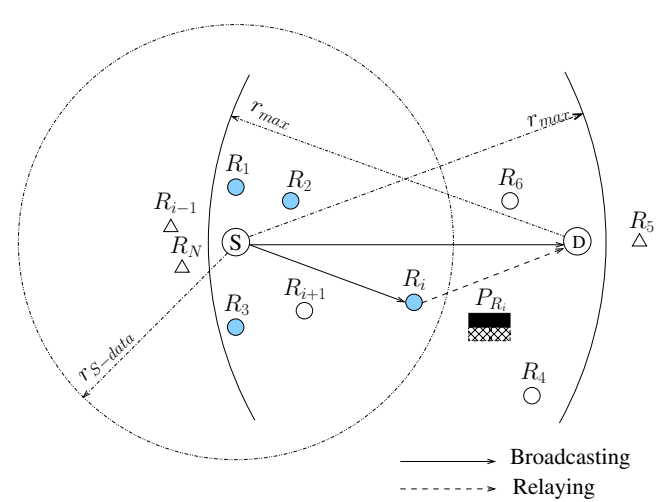

Fig. 1. The cooperative topology consists of one source $\mathcal{S}$, one destination $\mathcal{D}$ and a total of $N$ relays $\mathcal{R}=\left\{\mathcal{R}_{1}, \ldots, \mathcal{R}_{N}\right\}$.

\section{B. MAC Protocol Description}

We further develop our previously conceived protocol of [2] into an auction-style relay selection and frame combining regime. Due to space limitations, we assume the knowledge of [2] and focus on highlighting our proposed improvements.

Following the Request-To-Send (RTS) / Clear-To-Send (CTS) signalling exchange of the IEEE 802.11 protocol, which relies on the maximum transmit power, $\mathcal{S}$ broadcasts its data frame at a reduced power of $P_{\mathcal{S}-\text { data }}$, simultaneously indicating its throughput requirement of $\alpha C_{\mathcal{S}, \mathcal{D}}^{\max }(\alpha \geq 1)$, where $\alpha$ is the ratio of the desired and affordable throughput termed as 'the factor of greediness'. The factor $\alpha$ is adjusted by $\mathcal{S}$ to achieve further throughput improvements, which in fact may exceed the maximum throughput of the Source-to-Destination (SD) link, namely $C_{\mathcal{S}, \mathcal{D}}^{\max }$ which would be achievable in the absence of relaying. When $\alpha$ is higher than unity, the source data cannot be successfully transmitted to $\mathcal{D}$. However, $\mathcal{D}$ will store this data frame and combines it with the next data frame, in order to achieve an increased aggregated throughput.

Let $\mathcal{R}_{c}$ be a potential cooperative relay set. If a cooperative relay $\mathcal{R}_{i} \in \mathcal{R}_{c}$ receives a data frame from $\mathcal{S}$ correctly, it calculates the transmit power $P_{\mathcal{R}_{i}}^{\mathcal{S}}$ required for satisfying the throughput requirement of the source, namely $\alpha C_{\mathcal{S}, \mathcal{D}}^{\max }$. Being naturally selfish, $\mathcal{R}_{i}$ also reserves a certain fraction of $\beta C_{\mathcal{R}_{i}, \mathcal{D}}^{\max }(0<\beta<1)$ of the Relay-to-Destination (RD) channel capacity for conveying its own traffic, where $\beta$ is the relay's factor of greediness. Hence, $\mathcal{R}_{i}$ has to determine the specific transmit power $P_{\mathcal{R}_{i}}^{\mathcal{R}}$ required for guaranteeing a throughput of $\beta C_{\mathcal{R}_{i}, \mathcal{D}}^{\max }$. The source's and relay's data is transmitted concurrently with the aid of superposition coding [8]. If the total transmit power $P_{\mathcal{R}_{i}}=P_{\mathcal{R}_{i}}^{\mathcal{S}}+P_{\mathcal{R}_{i}}^{\mathcal{R}}$ required for successfully sending the data of both $\mathcal{S}$ and $\mathcal{R}_{i}$ to $\mathcal{D}$ does not exceed the maximum transmit power $P_{\max }$, then $\mathcal{R}_{i}$ would participate in the competition amongst the $N$ relays. Hence $\mathcal{R}_{i}$ submits $P_{\mathcal{R}_{i}}$ to $\mathcal{S}$ within its Relay-Request-To-Send (RRTS) message. In turn, $\mathcal{S}$ responds to the winning relay $\mathcal{R}_{i}$, who promises to consume the lowest transmit power by sending a Please-Send (PS) message, as shown in Fig 2.

If none of the RNs compete for a transmission opportunity, $\mathcal{S}$ directly sends its data to $\mathcal{D}$ as a replica without relaying, either at the specific transmit power, which is capable of guaranteeing the expected throughput of $\alpha C_{\mathcal{S}, \mathcal{D}}^{\max }$ or failing that, it resorts to the maximum transmit power $P_{\max }$.

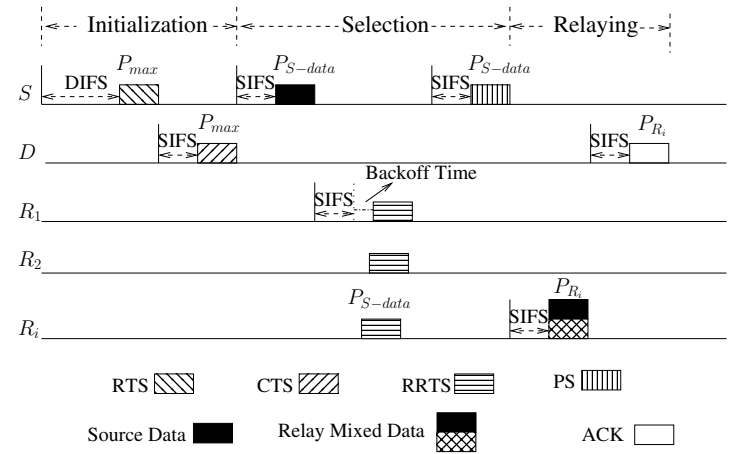

Fig. 2. The overall signalling procedure. RTS: Request-To-Send; CTS: ClearTo-Send; RRTS: Relay-Request-To-Send; PS: Please-Send; ACK: Acknowledgment; DIFS: Distributed Interframe Space; SIFS: Short Interframe Space.

\section{Auction-Style Relay Selection}

An auction entails the buying and selling of products. An auctioneer conducts the auction, while a set of bidders are the potential buyers who make the bids. The auction mechanisms determine the allocation rule and the payment rule [9]. Based on the first-price sealed-bid auction, we design a cooperative negotiation regime for selecting the best relay.

Analogously, we might argue that the source $\mathcal{S}$ and the $N$ relays act as the auctioneer and bidders, respectively. The transmit power $P_{\mathcal{R}_{i}}$ of bidder $\mathcal{R}_{i}$ offered for the sake of successfully transmitting both the source's and the relay's data constitutes a bid. In their capacity, as bidders, the relays submit their sealed bids to $\mathcal{S}$ in order to compete for a transmission opportunity to convey their data in the buffer. With the aid of this negotiation, $\mathcal{S}$ may improve both its throughput and its energy efficiency by accepting the winning relay's superposition coded simultaneous transmission in exchange for the relay's cooperation.

1) Utility Function: We first analyze the aim of the bidders. Relay $\mathcal{R}_{i}$ is interested in acquiring a transmission opportunity for power-efficiently conveying its traffic. Additionally, $\mathcal{R}_{i}$ also has to relay data for $\mathcal{S}$, in order to support the source's actions. Since $\mathcal{R}_{i}$ also intends to guarantee its own throughput requirement of $\beta C_{\mathcal{R}_{i}, \mathcal{D}}^{\max }$, an increased transmit power is required for conveying both superposition-coded data frames in comparison to that necessitated by sending only one of them. Hence, the goal of $\mathcal{R}_{i}$ is to serve both the source and itself, while minimizing total the transmit power. In order to reflect this goal of $\mathcal{R}_{i}$, we define the utility function as:

$$
\begin{aligned}
u_{i}= & {\left[P_{\text {max }}-P_{\mathcal{R}_{i}}\left(P_{\mathcal{R}_{i}}^{\mathcal{S}}, P_{\mathcal{R}_{i}}^{\mathcal{R}} \mid \alpha, \beta\right)\right] \cdot \delta(x) } \\
x= & \left\{P_{\mathcal{R}_{i}}\left(P_{\mathcal{R}_{i}}^{\mathcal{S}}, P_{\mathcal{R}_{i}}^{\mathcal{R}} \mid \alpha, \beta\right)=\right. \\
& \left.\min \left[P_{\text {max }}, P_{\mathcal{R}_{k}}\left(P_{\mathcal{R}_{k}}^{\mathcal{S}}, P_{\mathcal{R}_{k}}^{\mathcal{R}} \mid \alpha, \beta\right)_{k=1}^{n}\right]\right\},
\end{aligned}
$$

where $n$ denotes the number of bidders, while the indicator function $\delta(x)$ equals to unity, when its argument is satisfied. Otherwise, the value of $\delta(x)$ is zero. Eq (1) shows that if relay $\mathcal{R}_{i}$ wins the competition amongst all the $n$ contending relays, it obtains a utility of $u_{i}=\left(P_{\max }-P_{\mathcal{R}_{i}}\right)$. Otherwise its utility is zero. Hence, in the spirit of $\mathrm{Eq}(1), \mathcal{R}_{i}$ has to minimize its transmit power $P_{\mathcal{R}_{i}}$, if it intends to maximize its utility.

2) Allocation Rule: Upon registering the relays' intention to cooperate, $\mathcal{S}$ selects the winning $\mathcal{R}_{\hat{i}}$ according to the 
allocation rule advocated, which can be formulated as:

$$
\begin{aligned}
\hat{i}= & \arg \min _{i} P_{\mathcal{R}_{i}}\left(P_{\mathcal{R}_{i}}^{\mathcal{S}}, P_{\mathcal{R}_{i}}^{\mathcal{R}} \mid \alpha, \beta\right) . \\
& \delta\left[P_{\mathcal{R}_{i}}\left(P_{\mathcal{R}_{i}}^{\mathcal{S}}, P_{\mathcal{R}_{i}}^{\mathcal{R}} \mid \alpha, \beta\right) \leq P_{\text {max }}\right],
\end{aligned}
$$

subject to the condition $C_{\mathcal{S}}=\alpha C_{\mathcal{S}, \mathcal{D}}^{\max }(\alpha>1)$ and $C_{\mathcal{R}_{i}}^{\mathcal{R}} \geq$ $\beta C_{\mathcal{R}_{i}, \mathcal{D}}^{\max }(0<\beta<1)$, where $\hat{i}$ is the index of the winning relay, while $C_{\mathcal{S}}$ denotes the target throughput of $\mathcal{S}$. The variable $C_{\mathcal{R}_{i}}^{\mathcal{R}}$ represents the reserved throughput of $\mathcal{R}_{i}$. According to Eq (2), the specific relay requiring the lowest transmit power will be selected by $\mathcal{S}$.

3) Payment Rule: Similarly to the first-price sealed-bid auction, the winning relay $\mathcal{R}_{\hat{i}}$ is required to pay for its transmission opportunity, as part of the proposed negotiation. Thus we define the payment rule as:

$$
\hat{P}_{\mathcal{R}_{\hat{i}}}= \begin{cases}P_{\mathcal{R}_{\hat{i}}}\left(P_{\mathcal{R}_{\hat{i}}^{\mathcal{S}}}^{\mathcal{S}}, P_{\mathcal{R}_{\hat{i}}}^{\mathcal{R}} \mid \alpha, \beta\right) & \hat{i}=1, \ldots, n \\ \min \left(P_{\mathcal{S}, \mathcal{D}}^{(2)}, P_{\text {max }}\right) & \hat{i}=0 .\end{cases}
$$

When $\mathcal{S}$ does not receive any 'intention to cooperate' message from the relays, the process outcome becomes $\left(\hat{i}, \hat{P}_{\mathcal{R}_{\hat{i}}}\right)=$ $\left[0, \min \left(P_{\mathcal{S}, \mathcal{D}}^{(2)}, P_{\max }\right)\right]$. This implies that $\mathcal{S}$ will retransmit its data to $\mathcal{D}$ at the transmit power of $P_{S D}^{(2)}$, which is sufficiently high for achieving the source's target throughput upon combining the message with that of the most recent direct transmission, provided that $P_{\mathcal{S}, \mathcal{D}}^{(2)}$ is lower than $P_{\max }$. Otherwise, $\mathcal{S}$ assigns $P_{\max }$ to this transmission.

4) Relaying Strategy: According to the above resource allocation rule, $\mathcal{R}_{i}$ must use its minimum transmit power $P_{\mathcal{R}_{i \text { min }}}$ in order to win a transmission opportunity. Hence, the relays are motivated to employ the minimum power $P_{\mathcal{R}_{i}^{\text {min }}}^{\mathcal{R}}$, when aiming for achieving their desired throughput of $\beta C_{\mathcal{R}_{i}, \mathcal{D}}^{\max }$. Therefore, the relay's strategy is formulated as:

$$
\left(P_{\mathcal{R}_{i}}^{\mathcal{S}}, P_{\mathcal{R}_{i}^{m i n}}^{\mathcal{R}} \mid \alpha, \beta\right)=\arg \min _{P_{\mathcal{R}_{i}}^{\mathcal{R}}} P_{\mathcal{R}_{i}}\left(P_{\mathcal{R}_{i}}^{\mathcal{S}}, P_{\mathcal{R}_{i}}^{\mathcal{R}} \mid \alpha, \beta\right),
$$

subject to the condition of $C_{\mathcal{S}}=\alpha C_{\mathcal{S}, \mathcal{D}}^{\max }(\alpha>1)$ and $C_{\mathcal{R}_{i}}^{\mathcal{R}}=$ $C_{\mathcal{R}_{i}, \mathcal{D}}^{\max }(0<\beta<1)$, where we have:

$$
P_{\mathcal{R}_{i}}\left(P_{\mathcal{R}_{i}}^{\mathcal{S}}, P_{\mathcal{R}_{i}}^{\mathcal{R}} \mid \alpha, \beta\right)=P_{\mathcal{R}_{i}}^{\mathcal{S}}+P_{\mathcal{R}_{i}}^{\mathcal{R}} .
$$

Finding $P_{\mathcal{R}_{i}}^{\mathcal{S}}$ and $P_{\mathcal{R}_{i}^{\text {min }}}^{\mathcal{R}}$ of Eq (5) as well as the details of our received frame combining techniques are elaborated next.

\section{Frame Combining Technique}

In our design, the relay employs superposition coding [8] to encode both the source's and its own data. The destination then extracts the source's data from the superposition-coded relayed composite signal with the aid of Successive Interference Cancelation (SIC). Finally, the extracted relayed and the direct component are combined.

Before commencing our discussions on the frame combining technique, we formulate the maximum achievable rates of the SD and RD links as:

$$
C_{\mathcal{X}, \mathcal{D}}^{\max }=\log _{2}\left(1+\frac{\rho_{\mathcal{X}, \mathcal{D}}\left|h_{\mathcal{X}, \mathcal{D}}\right|^{2} P_{\max }}{P_{N}}\right) \quad \mathcal{X} \in\left\{\mathcal{S}, \mathcal{R}_{i}\right\}
$$

where $P_{N}$ is the power of the Additive White Gaussian Noise (AWGN), while $\left|h_{\mathcal{X}, \mathcal{D}}\right|$ denotes the magnitude of the flat Rayleigh channel between $\mathcal{X}$ and $\mathcal{D}$. Furthermore, $\rho_{\mathcal{X}, \mathcal{D}}$ denotes the free-space pathloss gain between $\mathcal{X}$ and $\mathcal{D}$.

As a first step, $\mathcal{D}$ extracts the source's data from the relay's superimposed data frame by treating the relay's data as interference. Hence, the source's achievable throughput is:

$$
C_{\mathcal{S}}=\log _{2}\left(1+\gamma_{\mathcal{S}, \mathcal{D}}^{(1)}+\gamma_{\mathcal{R}_{i}}^{\mathcal{S}}\right),
$$

where $\gamma_{\mathcal{S}, \mathcal{D}}^{(1)}$ denotes the receive Signal to Interference plus Noise Ratio (SINR) of the direct transmission in the first phase, which can be written as: $\gamma_{\mathcal{S}, \mathcal{D}}^{(1)}=$ $\rho_{\mathcal{S}, \mathcal{D}}\left|h_{\mathcal{S}, \mathcal{D}}\right|^{2} P_{\mathcal{S}-\text { data }} / P_{N}$. Furthermore, $\gamma_{\mathcal{R}_{i}}^{\mathcal{S}}$ represents the receive SINR of the source's data frame, which is transmitted during the relaying phase. More explicitly, when treating the relay's data frame as interference, $\gamma_{\mathcal{R}_{i}}^{\mathcal{S}}$ is given by:

$$
\gamma_{\mathcal{R}_{i}}^{\mathcal{S}}=\frac{\rho_{\mathcal{R}_{i}, \mathcal{D}}\left|h_{\mathcal{R}_{i}, \mathcal{D}}\right|^{2} P_{\mathcal{R}_{i}}^{\mathcal{S}}}{P_{N}+\rho_{\mathcal{R}_{i}, \mathcal{D}}\left|h_{\mathcal{R}_{i}, \mathcal{D}}\right|^{2} P_{\mathcal{R}_{i}}^{\mathcal{R}}} .
$$

After successfully retrieving the source's data frame, $\mathcal{D}$ becomes capable of decoding the relay's data frame by removing the source's interference with the aid of a SIC scheme. Hence, the achievable throughput of the relay may be formulated as:

$$
C_{\mathcal{R}_{i}}^{\mathcal{R}}=\log _{2}\left(1+\frac{\rho_{\mathcal{R}_{i}, \mathcal{D}}\left|h_{\mathcal{R}_{i}, \mathcal{D}}\right|^{2} P_{\mathcal{R}_{i}}^{\mathcal{R}}}{P_{N}}\right) .
$$

According to the relaying strategy employed, the relay calculates the minimum power required for the throughput formulated in Eq (9) to reach $\beta C_{\mathcal{R}_{i}, \mathcal{D}}^{\max }$. Thus, the value of $P_{\mathcal{R}_{i}^{\text {min }}}^{\mathcal{R}}$ is explicitly given as:

$$
P_{\mathcal{R}_{i}^{\min }}^{\mathcal{R}}=\frac{\left(2^{\beta C_{\mathcal{R}_{i}, \mathcal{D}}^{\max }}-1\right) P_{N}}{\rho_{\mathcal{R}_{i}, \mathcal{D}}\left|h_{\mathcal{R}_{i}, \mathcal{D}}\right|^{2}} \quad 0<\beta<1 .
$$

Likewise, based on Eq (7), Eq (8) and Eq (10), the relay is capable of calculating the transmit power $P_{\mathcal{R}_{i}}^{\mathcal{S}}$ required for successfully delivering the source's data at a throughput of $C_{\mathcal{S}}=\alpha C_{\mathcal{S}, \mathcal{D}}^{\max }$, which is given by:

$$
\begin{aligned}
& P_{\mathcal{R}_{i}}^{\mathcal{S}}=\gamma_{\mathcal{R}_{i}}^{\mathcal{S}}\left(\frac{P_{N}}{\rho_{\mathcal{R}_{i}, \mathcal{D}}\left|h_{\mathcal{R}_{i}, \mathcal{D}}\right|^{2}}+P_{\mathcal{R}_{i}^{\text {min }}}^{\mathcal{R}}\right) \\
& =\left(2^{\alpha C_{\mathcal{S}, \mathcal{D}}^{\max }}-\gamma_{\mathcal{S}, \mathcal{D}}^{(1)}-1\right) . \\
& {\left[\frac{P_{N}}{\rho_{\mathcal{R}_{i}, \mathcal{D}}\left|h_{\mathcal{R}_{i}, \mathcal{D}}\right|^{2}}+\frac{\left(2^{\beta C_{\mathcal{R}_{i}, \mathcal{D}}^{\max }}-1\right) P_{N}}{\rho_{\mathcal{R}_{i}, \mathcal{D}}\left|h_{\mathcal{R}_{i}, \mathcal{D}}\right|^{2}}\right],}
\end{aligned}
$$

subject to $\alpha>1$ and $0<\beta<1$. Provided that $\left|h_{\mathcal{R}_{i}, \mathcal{D}}\right|$, $\rho_{\mathcal{R}_{i}, \mathcal{D}}$ as well as $\gamma_{\mathcal{R}_{i}}^{\mathcal{S}}$ are known, which are calculated by the source from $\mathrm{Eq}(7)$ and are transmitted to $\mathcal{R}_{i}$ embedded into the source's data frame, $\mathcal{R}_{i}$ calculates $P_{\mathcal{R}_{i}^{\text {min }}}^{\mathcal{R}}$ and $P_{\mathcal{R}_{i}}^{\mathcal{S}}$ based on Eq (10) and Eq (11). Then $\mathcal{R}_{i}$ submits the value of $P_{\mathcal{R}_{i}^{\text {min }}}=P_{\mathcal{R}_{i}}^{\mathcal{S}}+P_{\mathcal{R}_{i}^{\text {min }}}^{\mathcal{R}}$ to the source as its bid.

Remarks: In general, superposition coding detected with the aid of SIC achieves different source/relay throughput combinations for the different possible decoding orders, although the resultant aggregated throughput remains the same. In our prefered decoding order, a lower source and a higher relay throughput is anticipated. Hence, as far as the source's throughput is concerned, the results provided in the next section may be interpreted as the lower bound. 


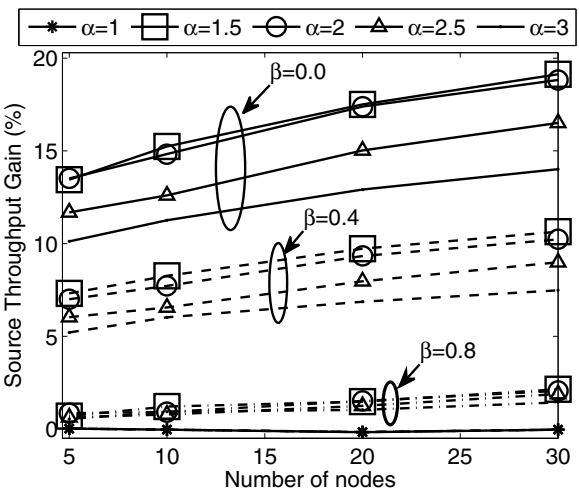

(a) The source's throughput gain

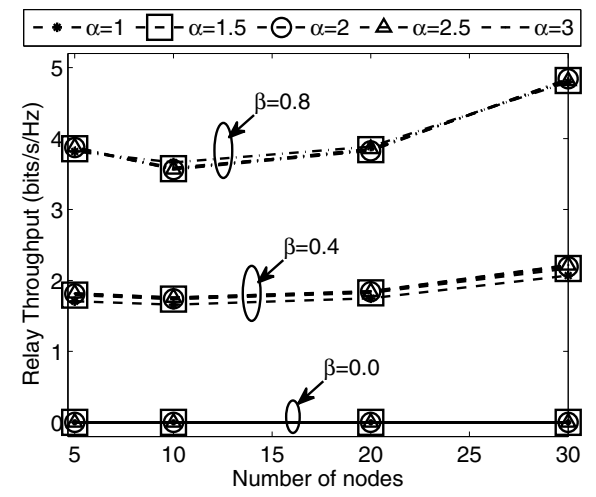

(b) The relay's throughput

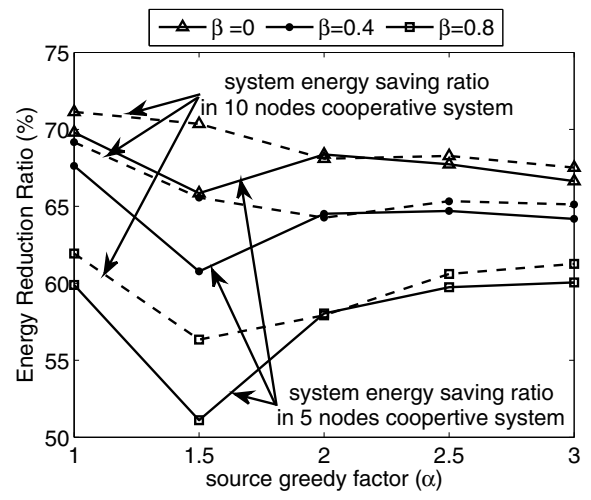

(c) Energy reduction ratio.

Fig. 3. (a) The source's throughput gain. (b) The relay's throughput. (c) The Energy reduction ratio.

\section{Simulation Results}

Our simulation results are based on using Omnet++. We consider the following two scenarios:

- In the first scenario, all the RNs are randomly distributed across the entire network area, while $\mathcal{S}$ and $\mathcal{D}$ have fixed positions. The network size considered ranges from $u=5$ nodes to $u=30$ nodes for the sake of evaluating the influence of the networks-size on the achievable throughput and energy efficiency.

- In the other scenario we consider a small network supporting $u=5$ nodes, i.e. $\mathcal{S}, \mathcal{D}$ and three RNs, where all the nodes have fixed positions. One of the three RNs is allocated at the normalized position of $d=1 / 4$ along the SD link. Another relay is in the middle of SD link at $d=1 / 2$, while the third relay is at point $d=3 / 4$ of the SD link. Given this scenario, let us now investigate the behavior of these three RNs.

Additionally, we also compare the attainable performance to that of a non-cooperative direct SD link, which has the same achievable source throughput corresponding to two direct transmission phases. In order to evaluate the performance, we adopt the idealized simplifying assumption that the control messages are received without errors. $P_{\max }$ and $P_{\mathcal{S}-\text { data }}$ are $2 m W$ and $1 m W$ respectively. The data packet length is 1024 Bytes.

\section{A. Achievable Throughput}

Compared to the maximum SD throughput of the noncooperative benchmarker, the source throughput gain of the cooperative regime relying on our cooperative MAC protocol is characterized in Fig 3(a). Observe in Fig 3(a) that as expected, the source throughput gain is inversely proportional to the factor of relay greediness $\beta$. When $\beta$ is equal to zero, the relay altruistically forwards the source's data frames. In this configuration, a source throughput gain in excess of $19 \%$ can be achieved by the cooperative regime, when $\alpha=1.5$ in a network hosting $u=30$ nodes. Given the same $\alpha$ and network size, our cooperative system is capable of providing a source throughput gain of $11 \%$ for $\beta=0.4$. When the relay becomes greedier and sets $\beta=0.8$, the source throughput still increases by about $2.5 \%$ compared to the maximum throughput of the SD channel operating in the direct non-cooperative regime. Under the constraint of $P_{\max }$, less relays are capable of affording the increased transmit power required, when $\beta$ is increased. This phenomenon reduces the probability of successful cooperative transmissions and erodes the benefits of the proposed MAC protocol. However, a considerable relay throughput improvement is offered by the proposed cooperative MAC protocol, as seen in Fig 3(b). The average throughput of the relay becomes $4.8 \mathrm{bit} / \mathrm{s} / \mathrm{Hz}$, for $\alpha=1.5$ when the number of nodes is $u=30$. When $\beta$ is 0.4 , the average throughput of the relay is still higher than $2 b i t / s / H z$ for the above network configuration.

As shown in Fig 3(a) and Fig 3(b), both the source's and relay's throughput increases gradually, when the network becomes larger. However, these trends are not as evident as those owing to the variations of $\alpha$ and $\beta$. Observe in Fig 3(b) that, the effect of the source's greediness $\alpha$ on the average relay-throughput is not as evident as that of $\beta$. However, the influence of the source's greediness $\alpha$ on the source's throughput gain is distinctly demonstrated in Fig 3(a). Apart from the configuration of $\alpha=1$, the source's throughput gain reduces, as $\alpha$ increases owing to having a reduced number of potential relays. Moreover, the difference between the source's throughput gains recorded for different values of $\alpha$ is reduced, when $\beta$ is increased. It also worth noting that the configuration of $\alpha=1.5$ always is the best for a give value of $\beta$. The above investigations imply that the proposed cooperative MAC protocol is capable of providing significant throughput improvements for both the source and relay. Considering the attainable throughput, observe in Fig 3(a) and Fig 3(b) that $\alpha=1.5$ is the best option for the source, since it offers the highest source throughput for each value of $\beta$, while guaranteeing a similar relay throughput compared to other configurations.

\section{B. Energy Consumption}

Fig 3(c) shows the achievable energy reduction ratio of $\frac{E_{\text {direct }}-E_{\text {coop }}}{E_{\text {direct }}} \times 100 \%$, where $E_{\text {coop }}$ denotes the energy consumption of our cooperative MAC protocol and $E_{\text {direct }}$ represents that of the direct non-cooperative two-slot transmission scheme, which is capable of achieving the same source throughput as the cooperative regime.

As seen in Fig 3(c), in excess of 50\% energy reduction is attained by exploiting the proposed protocol even in the 


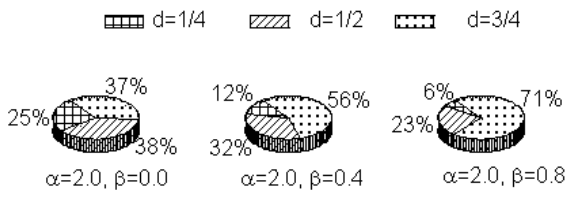

(a) Transmission probability of the relays

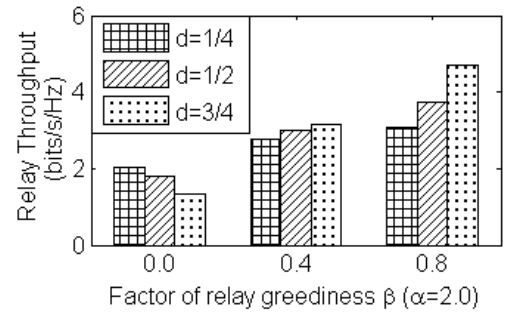

(b) relay throughput
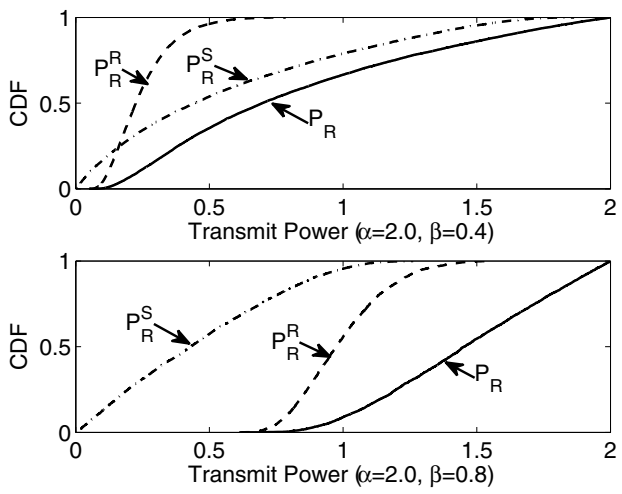

(c) The CDF of relay transmit power

Fig. 4. (a) and (b)The relay transmission probability and the throughput for each relay in a network hosting 5 nodes, namely $\mathcal{S}$, $\mathcal{D}$ and three RNs. (c) The CDF of the average relay transmit power $P_{\mathcal{R}}^{\mathcal{S}}$ required for conveying the SN's data, average relay transmit power $P_{\mathcal{R}}^{\mathcal{R}}$ assigned to its own data and the average total relay transmit power $P_{\mathcal{R}}$ in a network hosting $u=5$ nodes.

worst-case scenario, where we have $\alpha=1.5$ and $\beta=0.8$, respectively, while supporting $u=5$ nodes. As $\beta$ is increased, the system's energy reduction ratio drops from $70 \%$ to $56.3 \%$ for $\alpha=1.5$ and $u=10$. A reduced overall energy is consumed, when the number of nodes increases from $u=5$ to $u=10$. Since the curves for $u>10$ would overlap each other, we do not explicitly portray them in Fig 3(c) for the sake of avoiding obfuscating details. Based on the above analysis, the configuration of $\alpha=1.5$ provides a considerable throughput improvement for both the source and the relay, but also offers a satisfactory energy efficiency.

\section{Relay Behavior}

In order to investigate the behavior of relays, we analyze both the transmission probability and the achievable throughput of each relay as well as the Cumulative Distribution Function $(\mathrm{CDF})$ of the relays' transmit power for the configuration of $\alpha=2.0$ in the network hosting $u=5$ nodes.

Upon increasing $\beta$, the transmission probability of the relays at " $d=1 / 4 "$ and " $d=1 / 2 "$ decreases, while that of the relay at " $d=3 / 4 "$ increases, as shown in Fig 4(a). The relay at " $d=3 / 4$ " always benefits from the highest transmission probability for $\beta>0$ and the relay at " $d=1 / 4$ " benefits from the lowest probability of cooperative opportunities. As a benefit of its highest transmission probability, the relay at " $d=3 / 4$ " maintains the highest throughput, which is above $4.5 \mathrm{bits} / \mathrm{s} / \mathrm{Hz}$. The throughput of the relay at " $d=1 / 4$ " is lower than that of the relay at " $d=1 / 2$ ", as seen in Fig 4(b). However, when the three RNs altruistically dedicate to forwarding data frames for the source $(\beta=0)$, the throughput of the relay at " $d=1 / 4$ " which is allocated the lowest transmission probability is higher than that of the other relays. This implies that the relay at " $d=1 / 4$ " is capable of achieving considerable throughput improvements for each cooperative transmissions, although its transmission opportunities are more scarce than those of the relay at " $d=3 / 4 "$, which is close to the destination, when all the RNs are altruistic.

Fig 4(c) illustrates the CDF of the average relay transmit power $P_{\mathcal{R}}^{\mathcal{S}}=\mathbb{E}\left[P_{\mathcal{R}_{i}}^{\mathcal{S}}\right], \forall R_{i}$ required for conveying the source's data and that required for its own data transmission, namely
$P_{\mathcal{R}}^{\mathcal{R}}=\mathbb{E}\left[P_{\mathcal{R}_{i}}^{\mathcal{R}}\right], \forall R_{i}$, as well as that of the average relay transmit power of $P_{\mathcal{R}}=P_{\mathcal{R}}^{\mathcal{S}}+P_{\mathcal{R}}^{\mathcal{R}}$ for the configuration of $\alpha=2.0$ when $u=5$. When $\beta=0.4$, the relay assigns more transmit power for relaying the source's data frame. However, the transmit power $P_{\mathcal{R}}^{\mathcal{R}}$ assigned for transmitting the relay's data increases, when the relay becomes greedier. Indeed, $P_{\mathcal{R}}^{\mathcal{R}}$ becomes even higher than $P_{\mathcal{R}}^{\mathcal{S}}$, when $\beta=0.8$.

\section{CONCLUSIONS}

In this paper, a cooperative MAC layer protocol was proposed for striking a tradeoff between the attainable throughput and energy efficiency. When compared to its non-cooperative counterpart, the proposed scheme is capable of providing considerable throughput enhancements for both the source and relay, while achieving useful transmission energy savings.

\section{REFERENCES}

[1] F. Verde, T. Korakis, E. Erkip, and A. Scaglione, "A Simple Recruitment Scheme of Multiple Nodes for Cooperative MAC," IEEE Transactions on Communications, vol. 58, pp. 2667 - 2682, Sept 2010.

[2] J. Feng, R. Zhong, S. X. Ng, and L. Hanzo, "Relay Selection for EnergyEfficient Cooperative Media Access Control," in Wireless Communications and Networking Conference (WCNC), 2011 IEEE, Cancun, Mexico, March 2011, pp. 1-6.

[3] Z. Zhou, S. Zhou, J. Cui, and S. Cui, "Energy-Efficient Cooperative Communication Based on Power Control and Selective Single-Relay in Wireless Sensor Networks," IEEE Transactions on Communications, vol. 7, pp. 1536-1276, Aug 2008.

[4] L. Zhao, L. Guo, J. Zhang, and H. Zhang, "Game-Theoretic Medium Access Control Protocol for Wireless Sensor Networks," IET Communications, vol. 3, pp. 1274 - 1283, July 2009.

[5] G. N. Shirazi, P. Y. Kong, and C. K. Tham, "Optimal Cooperative Relaying Schemes in IR-UWB Networks," IEEE Transactions on Mobile Computing, vol. 9, pp. 969 - 981, July 2010.

[6] I. Stanojev, O. Simeone, U. Spagnolini, Y. Bar-Ness, and R. Pickholtz, "Cooperative ARQ Via Auction-Based Spectrum Leasing," IEEE Transactions on Communications, vol. 58, pp. 1843 - 1856, June 2010.

[7] A. Mukherjee and H. M. Kwon, "General Auction-Theoretic Strategies for Distributed Partner Selection in Cooperative Wireless Networks," IEEE Transactions on Communications, vol. 58, pp. 2903 - 2915, Oct 2010.

[8] T. M. Cover and J. A. Thomas, Elements of Information Theory. New Jersey, USA: WILEY-INTERSCIENCE, 2006.

[9] K. J. R. Liu and B. B. Wang, Cognitive Radio Networking and Security: A Game-Theoretic View. Cambridge, UK: Cambridge University Press, 2010 . 